\title{
Dehydrozingerone Exhibits Synergistic Antifungal Activities in Combination with Dodecanol against Budding Yeast via the Restriction of Multidrug Resistance
}

(요 $\odot \odot$

\author{
Authors \\ Chika Yamawaki', Yoshihiro Yamaguchi1,2, Akira Ogita1,3, Toshio Tanaka', Ken-ichi Fujita1
}

\section{Affiliations}

1 Graduate School of Science, Osaka City University, Sumiyoshi-ku, Osaka, Japan

2 Advanced Research Institute for Natural Science and Technology, Osaka City University, Sumiyoshi-ku, Osaka, Japan

3 Research Center for Urban Health and Sports, Osaka City University, Sumiyoshi-ku, Osaka, Japan

\section{Key words}

$A B C$ transporter, antifungal, dehydrozingerone, feruloylmethane, multidrug resistance, $P D R 5$, Saccharomyces cerevisiae, vanillylidenacetone

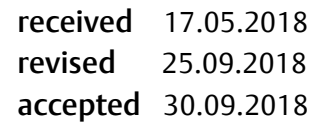

Bibliography DOI https://doi.org/10.1055/a-0757-7991

Planta Med Int Open 2018; 5: e61-e67

(c) Georg Thieme Verlag KG Stuttgart · New York

ISSN 2509-9264

Correspondence

Dr. Ken-ichi Fujita

Graduate School of Science

Osaka City University

3-3-138 Sugimoto

Sumiyoshi-ku

558-8585 Osaka

Japan

Tel.: + 81/6/6605 2580, Fax: + 81/6/6605 3164

kfujita@sci.osaka-cu.ac.jp

\begin{abstract}
Drug resistance in fungal infections has been a more frequent occurrence with the increasing number of immunocompromised patients. In efforts to overcome the problem of fungal drug resistance, we focused on the phenolic compound dehydrozingerone, which is isolated from Zingiber officinale. The effectiveness of this compound on the model yeast Saccharomyces cerevisiae has not been reported. In our study, dehydrozingerone showed a weak antifungal activity against the yeast, but demonstrated a synergistic effect in combination with dodecanol, which typically only restricts cell growth transiently. Efflux of rhodamine 6G through the multidrug efflux pumps was significantly restricted by dehydrozingerone. The transcription level of $P D R 5$, encoding a primary multidrug efflux pump in S. cerevisiae, was enhanced with dodecanol treatment, whereas the level was reduced by dehydrozingerone. These results suggest that dehydrozingerone may be effective for potentiating antifungal activity of other drugs that are expelled from fungi by multidrug transporters like Pdr5p.
\end{abstract}

\section{Abbreviations}

ABC ATP-binding cassette

CFU colony forming unit

DCF 2',7'-dichlorofluorescein

DCFH-DA 2', 7'-dichlorofluorescein diacetate

MFC minimum fungicidal concentration
MIC minimum growth inhibitory concentration

qRT-PCR real-time quantitative reverse transcriptase-polymerase chain reaction

R6G rhodamine 6G

RNA ribonucleic acid

ROS reactive oxygen species

YPD yeast extract peptone dextrose 


\section{Introduction}

With the increasing number of immunocompromised patients, fatal deep-seated mycoses frequently occur due to opportunistic invasive fungal infections [1]. Fungi and humans are both eukaryotic organisms. Because both are eukaryotes, the number of targets for antifungal drugs like ergosterol, the fungal cell wall, and cytosine deaminase has been limited due to their common or similar metabolisms and cellular structures [2]. Therefore, it is difficult to develop antifungals with fewer adverse effects and new modes of action.

Adding to the problem is the development of drug resistance. Clinical isolates have been reported to show resistance to antifungals such as azoles, including fluconazole [3] and 5-fluorocytosine [4]. The occurrence of pathogenic Candida clinical isolates with lower susceptibility to echinocandins has also been reported [5]. Therefore, strategies for overcoming drug resistance are needed in order to improve antifungal chemotherapy.

Mechanisms of drug resistance are generally classified as enzymatic degradation of the antifungals, the inability of the antifungals to bind to target sites due to mutation of their genes, or expulsion of antifungals to the extracellular space [6]. Among these, fungi have particularly developed various multidrug efflux pumps such as ATP-dependent ABC transporters that expel harmful drugs from the fungi [7-9].

The polyphenol curcumin ( $\vee$ Fig. 1) is a main constituent of turmeric isolated from the rhizomes of Curcuma longa L., a member of the ginger family (Zingiberaceae). Curcumin is reported to reverse the effects of multidrug resistance in human colon carcinoma, human gastric carcinoma, and human osteosarcoma cell lines [10-12]. Furthermore, curcumin is reported to modulate drug efflux in the nonpathogenic model yeast Saccharomyces cerevisiae cells overexpressing the $\mathrm{ABC}$ transporter Pdr5p, and the Candida albicans $A B C$ transporters $C d r 1 p$ and $C d r 2 p$ [13]. Even with the assistance of surfactants, curcumin is difficult to dissolve in waterbased solutions because of its high hydrophobicity. Thus, this compound seems to be unsuitable for use in clinical applications in addition to food protection.

In order to improve drug solubility in water, we selected, as a promising new candidate, the phenolic compound dehydrozingerone [(E)-4-(4-hydroxy-3-methoxyphenyl)but-3-en-2-one] (synonyms, feruloylmethane, and vanillylidenacetone; $>$ Fig. 1). Dehy-

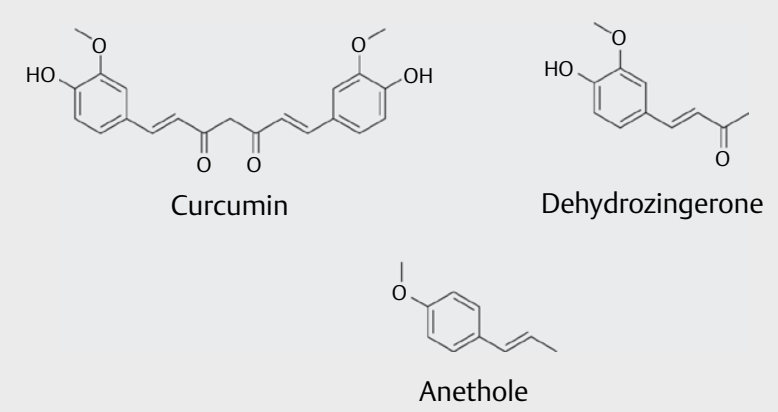

- Fig. 1 Chemical structures of curcumin, dehydrozingerone, and trans-anethole. drozingerone is a water-soluble and half structural analog of curcumin ( $\triangleright$ Fig. 1), and a pungent constituent in the rhizomes of ginger Zingiber officinale Roscoe (Zingiberaceae) [14]. On the other hand, the phenylpropanoid trans-anethole ( $\mathbf{F i g . ~ 1 ) , ~ a ~ p r i n c i p a l ~}$ constituent of anise oil, demonstrates synergistic and durable antifungal effects against $S$. cerevisiae via its induced inhibition of gene expression of the fungal multidrug efflux pumps, primarily Pdr5p [15]. Therefore, phenols, including dehydrozingerone, in addition to curcumin, in which phenylpropanoid-like structures are involved, were also expected to show such an effect. Dehydrozingerone has been reported to show various biological activities, including antitumor, antioxidative, anti-amyloid, and anti-inflammatory activities $[14,16]$. In addition, its antifungal activities against Aspergillus oryzae, Aspergillus flavus, Aspergillus niger, Aspergillus ochraceus, Fusarium oxysporum, and Penicillium chrysogenum have also been reported [17]. However, its antifungal potency against these fungi were weaker than the antifungal agents that were already available on the market.

Since it did show some antifungal activity, dehydrozingerone was expected to show synergistic and potentially durable antifungal activity when combined with other drugs due to its ability to restrict drug efflux from the fungi. In the current study, we evaluated the effects of dehydrozingerone with the antifungal action of the model antifungal agent $n$-dodecanol, which transiently restricts fungal growth [15]. In addition, we investigated the function of drug efflux, and the gene expression of PDR5, a primary multidrug efflux pump in S. cerevisiae, which is upregulated by dodecanol [15]. Findings from these studies and the structure-activity relationships of dehydrozingerone as a lead compound may be beneficial in developing new strategies for overcoming drug resistance and improving antifungal chemotherapy.

\section{Results}

We here examined the effects of dehydrozingerone and dodecanol, separately and in combination, on the growth of S. cerevisiae based on CFU measurements ( $>$ Fig. 2). The MIC of dehydrozingerone was greater than $4000 \mu \mathrm{M}$ against S. cerevisiae ( $\triangleright$ Table 1 ). Its efficacy was less than that of the phenylpropanoid trans-anethole ( $\triangleright$ Table 1 ). Treatment with $2000 \mu \mathrm{M}$ dehydrozingerone failed to show any definitive fungicidal activity against the yeast cells, and demonstrated only weak inhibitory effects on fungal growth in a time-kill assay ( Fig. 2). On the other hand, dodecanol is reported to show a rapid fungicidal effect against $S$. cerevisiae, but its effect is not long lasting, with only transient activity [15]. The MIC of dodecanol was $40 \mu \mathrm{M}$ against $\mathrm{S}$. cerevisiae at $24 \mathrm{~h}$ post-drug exposure. At post-drug exposure times beyond $24 \mathrm{~h}$, the antifungal activity of dodecanol was not observed (> $2000 \mu \mathrm{M}$; $>$ Table 1 ). Rapid reduction and restoration of cell viability were observed within $24 \mathrm{~h}$ of drug exposure with $156 \mu \mathrm{M}$ dodecanol, indicating the transient fungicidal activity induced by the fatty alcohol ( $>$ Fig. 2). However, beyond $48 \mathrm{~h}$ incubation, viability was recovered to levels seen in the control cells. These results suggested that dodecanol was unable to induce a fungicidal effect following a long period after drug exposure. However, cell viability was almost completely inhibited $72 \mathrm{~h}$ after drug exposure when the cells were treated with $2000 \mu \mathrm{M}$ dehydrozingerone in 


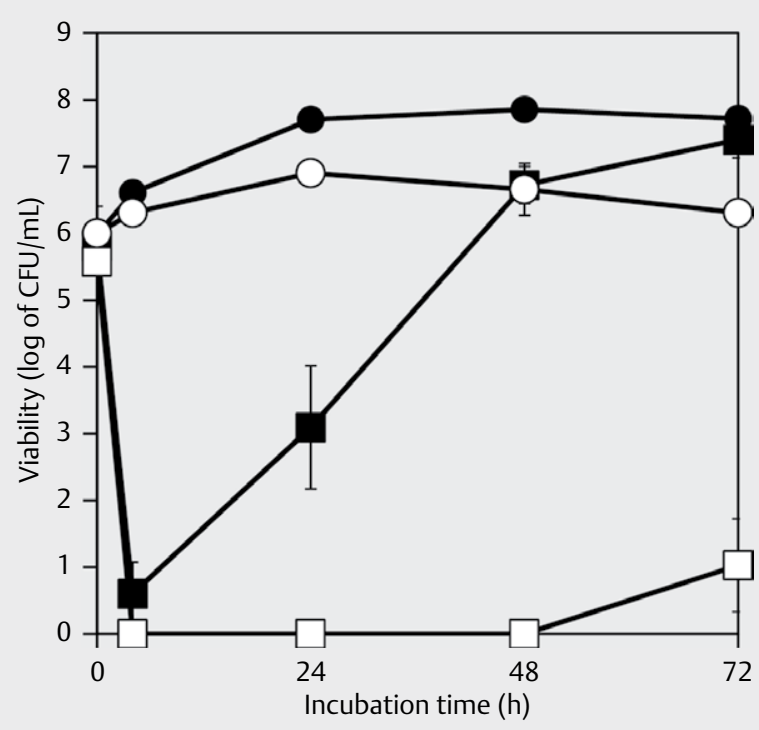

- Fig. 2 Effect of dehydrozingerone on dodecanol-induced temporary cell death of $S$. cerevisiae BY4741. Yeast cells were grown in YPD medium at $30^{\circ} \mathrm{C}$. Drugs were added to the culture as follows: none (closed circle), $156 \mu \mathrm{M}$ dodecanol (closed square), $2000 \mu \mathrm{M}$ dehydrozingerone (open circle), or $156 \mu \mathrm{M}$ dodecanol $+2000 \mu \mathrm{M}$ dehydrozingerone (open square). Data are means \pm standard deviations of 3 independent experiments.

Table 1 MICs of anethtole, dodecanol, and dehydrozingerone in S. cerevisiae.

\begin{tabular}{|l|c|}
\hline Compounds & MIC $(\boldsymbol{\mu M})$ \\
\hline Anethole & 1250 \\
\hline Dodecanol & $>2000$ \\
\hline Dehydrozingerone & $>4000$ \\
\hline
\end{tabular}

combination with $156 \mu \mathrm{M}$ dodecanol ( $\vee$ Fig. 2). In addition, synergistic MFCs were also observed at $31.25 \mu \mathrm{M}$ dodecanol and $500 \mu \mathrm{M}$ dehydrozingerone. These results suggested that the dehydrozingerone sustained the transient fungicidal effect on S. cerevisiae from dodecanol alone.

We examined whether dehydrozingerone could restrict glucoseinduced R6G efflux in S. cerevisiae. When the cells were treated without dehydrozingerone, the fluorescent intensity derived from R6G exhausted into supernatants was elevated linearly with increased incubation times ( $\mathbf{F i g . ~ 3 ) . ~ I n ~ c o m p a r i s o n , ~ w h e n ~ t h e ~ y e a s t ~ w a s ~}$ treated with $2000 \mu \mathrm{M}$ dehydrozingerone, the level of fluorescence decreased by $58.5 \%$ compared with the control cells that were not exposed to the drug. These results suggested that dehydrozingerone significantly restricted the drug efflux, possibly being depend on glucose-stimulated multidrug efflux pumps. Therefore, we next examined the effects of dehydrozingerone on the expression of genes related to the multidrug efflux pumps.

The expression of $P D R 5$, which encodes a primary multidrug efflux pump in $S$. cerevisiae, resulted in dodecanol being removed from the cell [15]. On the other hand, PDR5 may be suppressed by

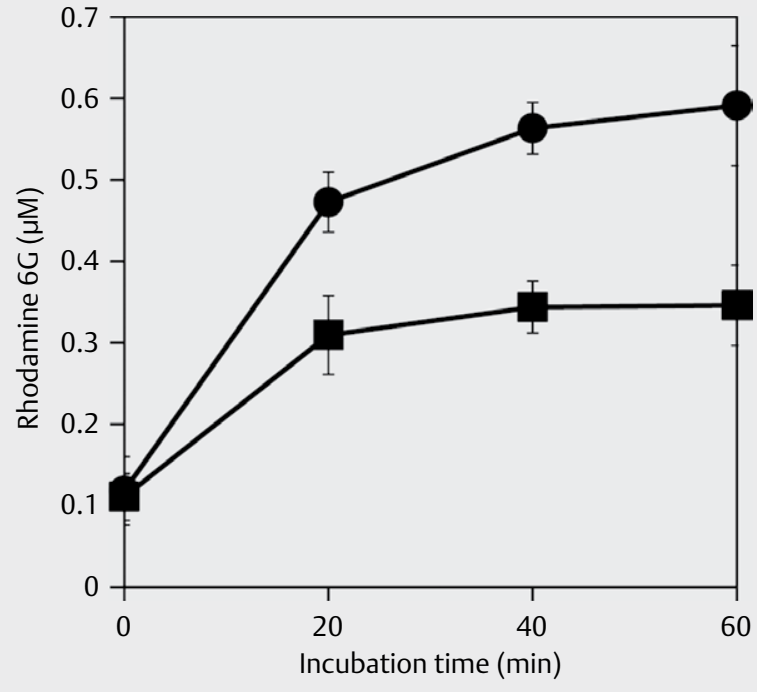

- Fig. 3 Effect of dehydrozingerone on the drug efflux of R6G. Yeast cells were incubated without shaking at $30^{\circ} \mathrm{C}$ in PBS containing $10 \mathrm{mM}$ glucose with $2000 \mu \mathrm{M}$ dehydrozingerone (closed square) or without dehydrozingerone (closed circle). The fluorescence intensity of the supernatant was measured using a GENios microplate reader with $485 \mathrm{~nm}$ excitation and $535 \mathrm{~nm}$ emission filters. Data are means \pm standard deviations of 3 independent experiments.

dehydrozingerone in a fashion similar to that observed with curcumin treatment. Based on qRT-PCR analysis, when the cells were treated with $2000 \mu \mathrm{M}$ dehydrozingerone, the transcription levels of PDR5 were similar to those seen in control cells not treated with dehydrozingerone ( $\triangleright$ Fig. 4). When the cells were treated with $156 \mu \mathrm{M}$ dodecanol, the PDR5 transcription levels were elevated approximately 4.5 -fold above that observed in non-treated cells. However, in treatment of the yeast with the combination of $156 \mu \mathrm{M}$ dodecanol and $2000 \mu \mathrm{M}$ dehydrozingerone, a $45 \%$ reduction was observed in the transcription level compared with the dodecanol only treatment. These results suggested that dehydrozingerone counteracted the activity of the drug efflux pumps, thereby reducing the intracellular levels of dodecanol by also suppressing the increased expression of PDR5 that dodecanol stimulated. As a result, the lack of recovery in cell viability was probably maintained long term after the exposure to dodecanol.

Next, we measured the transcription levels of PDR 1 and $P D R 3$, which are transcription factors of PDR5 [18], in the cells treated with dodecanol and/or dehydrozingerone. Pdr1p encoded by PDR 1 responds to intracellular stress signals, after which it promotes the transcription of PDR3 [19]. Conversely, Pdr3p encoded by PDR3 regulates its transcription and that of $P D R 5[19,20]$. It was noted that the expression levels of PDR1,PDR3, and PDR5 were unaffected by dehydrozingerone ( $\triangleright$ Fig. 4). Conversely, the expression levels of PDR1 and PDR3 in the cells were approximately 1.7- and 6.1-fold, respectively, higher after treatment with $156 \mu \mathrm{M}$ dodecanol than they were without drug treatment. However, the combination of dehydrozingerone and dodecanol reduced the expression levels of 

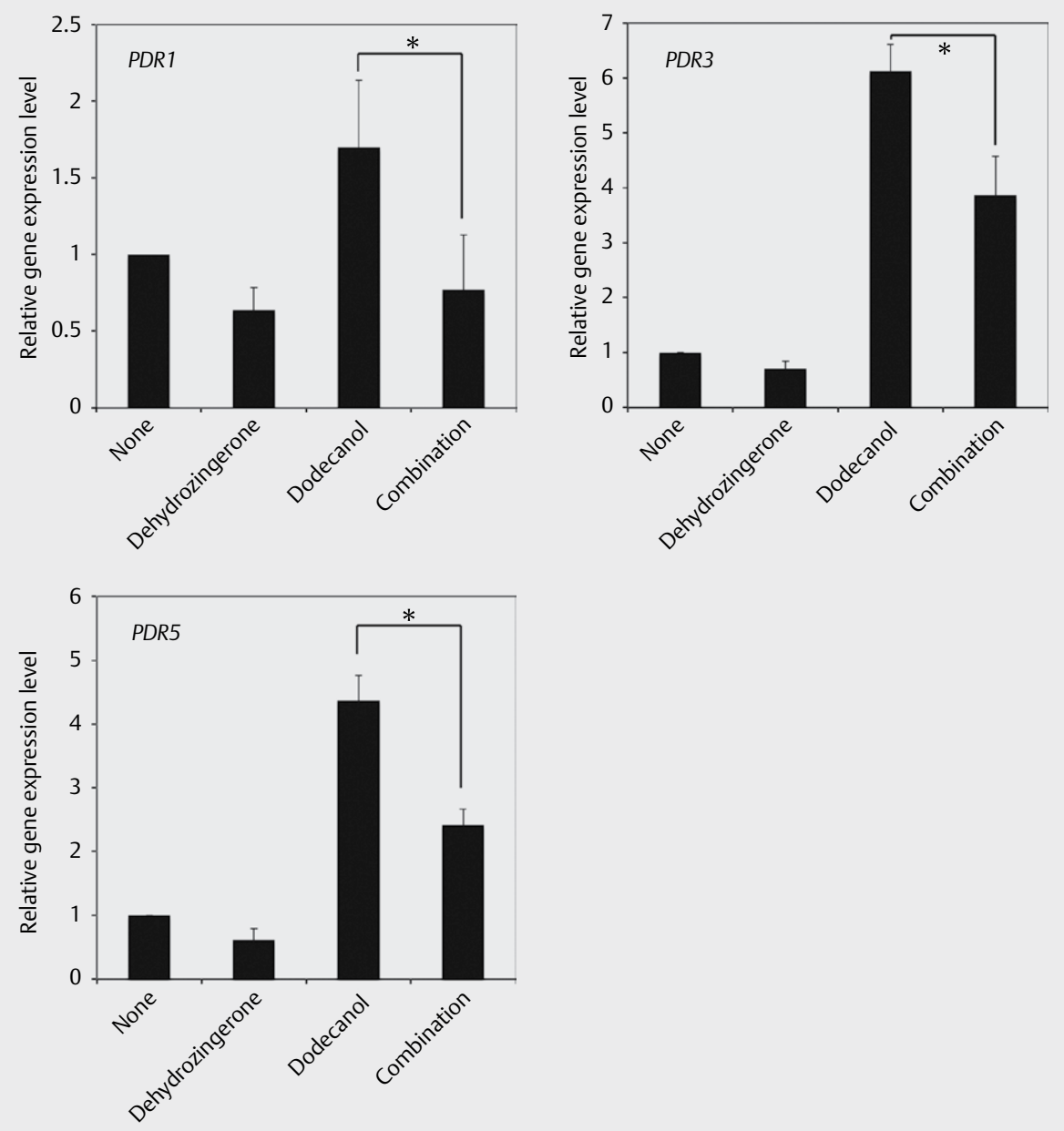

- Fig. 4 Relative gene expression levels for PDR1, PDR3, and PDR5 normalized to ACT1. S. cerevisiae cells were incubated in YPD medium containing $2000 \mu \mathrm{M}$ dehydrozingerone, $156 \mu \mathrm{M}$ dodecanol, or both prior to total RNA extraction and qRT-PCR analysis. Data are means \pm standard deviations of 3 independent experiments $\left({ }^{*} p<0.05\right)$.

PDR 1 and PDR3 by 45 and $63 \%$ compared to dodecanol-treated cells, respectively. These results suggest that dehydrozingerone prevents dodecanol-induced overexpression of PDR 1 and PDR3 in addition to $P D R 5$. This indicates that dehydrozingerone possibly maintains the accumulation of dodecanol in the cells, thereby preventing the restoration of cell viability. However, it is unclear whether dehydrozingerone directly affects the transcription of PDR1, $P D R 3$, and $P D R 5$ or other genes.

The phenylpropanoid trans-anethole, a principal component of anise oil, is reported to exhibit synergistic antifungal activity against S. cerevisiae and C. albicans when combined with dodecanol and fluconazole, respectively [15]. This synergistic activity also is involved in the attenuation of gene expression related to multidrug efflux pumps [15]. Anethole is reported to induce the production of ROS against a nonpathogenic strain of $S$. cerevisiae and a strain of Aspergillus fumigatus that is pathogenic in humans [21]. In addition, dehydrozingerone induces cell-cycle arrest at the G2/M phase, accompanying the accumulation of cellular ROS in the human colon cancer cell line HT-29 [22]. If dehydrozingerone induces cellular ROS pro- duction against fungi, including S. cerevisiae, the produced ROS may affect the expression of genes related to multidrug efflux. Therefore, we examined whether dehydrozingerone induced the production of cellular ROS. Hydrogen peroxide at a concentration of $2000 \mu \mathrm{M}$ greatly induced cellular ROS production ( $>$ Fig. 5). On the other hand, when the cells were treated with $2000 \mu \mathrm{M}$ dehydrozingerone, ROS production was not detected. This result suggested that decreased levels of gene expression, and R6G efflux caused by dehydrozingerone, were not dependent on ROS production.

\section{Discussion}

The MIC values of dehydrozingerone have been reported to be from 755 to $911 \mu \mathrm{M}$ against $A$. ochraceus, A. niger, A. flavus, A. oryzae, $P$. chrysogenum, and $F$. oxysporum [17]. The MFC values of this phenolic compound are from 880 to $1041 \mu \mathrm{M}$ against the same fungi described above [17]. Based on morphological alterations in hyphae and the subsequent cell lysis, the fungicidal activities may be a result of cell wall fragility [17]. Our study demonstrated a weak antifungal 


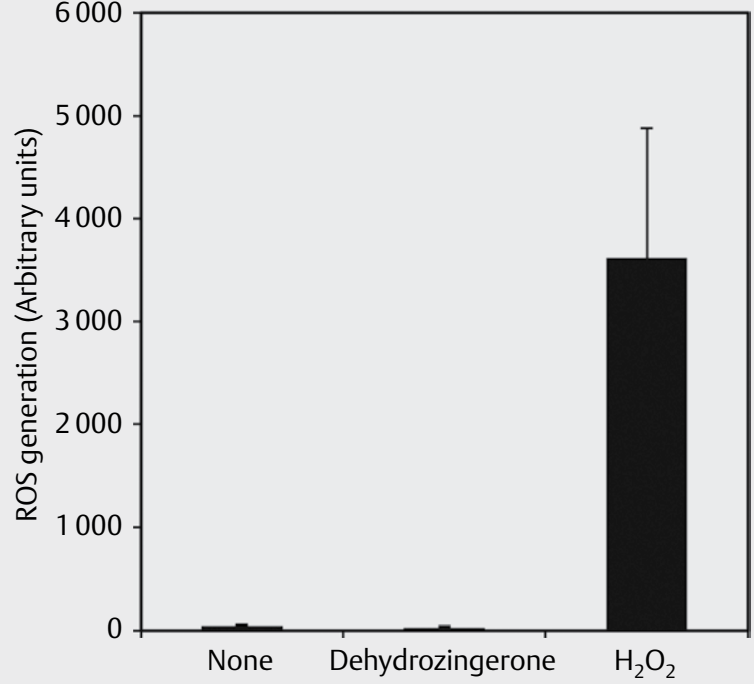

- Fig. 5 Effect of dehydrozingerone on ROS generation in S. cerevisiae cells. Exponentially growing $S$. cerevisiae cells were inoculated into $3 \mathrm{~mL}$ of YPD medium with or without $2000 \mu \mathrm{M}$ dehydrozingerone at $30^{\circ} \mathrm{C}$. Hydrogen peroxide $\left(\mathrm{H}_{2} \mathrm{O}_{2} ; 2 \mathrm{mM}\right)$ was used as a positive control. Data are means \pm standard deviations of 3 independent experiments.

activity of dehydrozingerone against the yeast S. cerevisiae, consistent with the findings for the filamentous fungi. The antifungal activity against S. cerevisiae (MIC > $4000 \mu \mathrm{M}$; $>$ Table 1) was weaker than that against other fungi previously reported [17]. Although we could not determine the distinct mechanism of the fungicidal activity, long-term exposure of dehydrozingerone in a nutrient medium induced a slight reduction of the cell viability that gradually increased following the cultivation of the cells in the presence of the drug ( $\triangleright$ Fig. 2). Generally, antimicrobials that affect the cell wall, and/or its metabolism, are accompanied with a decrease in cell viability and culture turbidity as a result of cell lysis [23, 24]. This may explain the harmful effect of dehydrozingerone on the cellular architecture of $S$. cerevisiae.

Dodecanol is a model drug in that it induces rapid and complete reduction in cell viability of S. cerevisiae, based on CFU analysis $[15,25]$. However, the reduction is only temporary. During a temporal analysis of treatment, the cell viability is gradually restored to that of cells not treated with drugs due to drug efflux [15]. When the $S$. cerevisiae cells were co-treated with dehydrozingerone, the transient fungicidal activity of dodecanol was converted to a longer lasting one ( $\triangleright$ Fig. 2). Curcumin is reported to inhibit drug efflux based on analyses using R6G assays in S. cerevisiae cells overexpressing Pdr5p and C. albicans ABC transporters Cdr1p and Cdr2p [13]. In addition, curcumin, in a dose-dependent fashion, reduced MDR 1mediated drug efflux in cervical carcinoma cells showing multidrug resistance via direct interaction between curcumin and MDR1 proteins, a primary human multidrug efflux pump [26]. Dehydrozingerone at $2000 \mu \mathrm{M}$, inducing a feeble growth inhibition ( $>$ Fig. 2), also inhibited R6G efflux ( $\triangleright$ Fig. 3), indicating that a half portion of curcumin was at least needed for the restriction of the drug efflux.
If dehydrozingerone decreases cytosolic levels of ATP as a result of its induced growth inhibition, R6G efflux, via ABC transporters driven by ATP, may be indirectly restricted.

Dodecanol is hypersensitive to a $S$. cerevisiae strain deficient in PDR5[15], a primary ABC transporter in S. cerevisiae for drug efflux [19]. Curcumin has been reported to regulate the mRNA expression of MDR 1 through inhibition of several signaling pathways related to phosphatidylinositol-4,5-bisphosphate 3-kinase, the serine/threonine protein kinase AKt, and nuclear factor-kappa B [27]. However, it is poorly understood whether curcumin directly binds to these proteins. Dehydrozingerone also restricted dodecanolinduced overexpression of PDR1, PDR3, and PDR5 ( $>$ Fig. 4), indicating that the inhibition of drug efflux caused by dehydrozingerone at least occurs at the level of gene transcription. The reduction of the expression levels in PDR1,PDR3, and PDR5 could not be explained only by cellular ATP depletion described above, as the expression levels of $A C T 1$, encoding the single essential gene for a cytoskeletal protein actin, were not affected by the treatment of dodecanol and/or dehydrozingerone (data not shown).

In our previous study showing synergetic antifungal activity, anethole inhibited drug efflux via transcriptional repression of several efflux pump genes [15]. In addition, anethole induces cellular ROS production [21]. Therefore, transcriptional repression may be involved in the ROS production. Dehydrozingerone did not induce ROS production ( $\triangleright$ Fig. 5), suggesting that the transcriptional repression was probably not triggered by ROS production. These results may be applicable to the case of curcumin in regards to its inhibition of drug efflux.

Curcumin is undesirable for use in clinical applications because it is difficult to dissolve in water-based solutions. On the other hand, dehydrozingerone, a water-soluble compound, is a potential candidate for the suppression of fungal proliferation in combination with other drugs in which the drug efficacy is tempered or abolished by multidrug resistance. However, high concentrations of dehydrozingerone (at least $2000 \mu \mathrm{M}$ ) were needed to exert such effects against $S$. cerevisiae. Therefore, it is difficult for dehydrozingerone to be directly applied to clinical use. Further studies on the structure-activity relationships of dehydrozingerone as a lead compound are needed for the enhancement of synergistic antifungal activities dependent on the restriction of multidrug resistance.

\section{Materials and Methods}

\section{Strains and culture conditions}

A parental strain S. cerevisiae BY4741 and its pdr5 knockout mutant were obtained from the Yeast Knockout Strain Collection (Thermo Scientific Open Biosystems). Yeast cells were grown in YPD medium containing $1 \%$ Bacto-yeast extract (Difco Laboratories), $2 \%$ Bacto-peptone (Difco Laboratories), and $2 \% \mathrm{D}$-glucose for $16 \mathrm{~h}$ at $30^{\circ} \mathrm{C}$ without shaking in preparation for experimental use.

\section{Chemicals}

n-Dodecanol (purity $97 \%$ by GC) was purchased from Kishida Chemical. trans-Anethole (purity $>98.0 \%$ by GC) and dehydrozingerone (purity $>98.0 \%$ by GC) were purchased from Tokyo Chemical Industry. DMF (purity $>99.0 \%$ by GC) was purchased from Wako pure 
chemicals. R6G (purity 99.0\%) and DCFH-DA (purity >97.0\%) were purchased from Sigma-Aldrich. Anethole, dodecanol, and dehydrozingerone were diluted with DMF, and R6G and DCFH-DA were diluted with ethanol prior to use.

\section{Antifungal assay}

Antifungal assays were performed using previously described methods [28, 29]. In general, serial 2-fold dilutions of the tested compounds were prepared in DMF, and $30 \mu \mathrm{L}$ of the 100 -fold diluted concentration were added to $3 \mathrm{~mL}$ of YPD medium in a test tube (diameter $10 \mathrm{~mm}$ ). The yeast cells were inoculated into the medium to give a final concentration of $10^{6} \mathrm{CFU} / \mathrm{mL}$. The cultures were incubated without shaking for $48 \mathrm{~h}$ at $30^{\circ} \mathrm{C}$. The MIC was determined as the lowest concentration of test compound at which no visible fungal growth was detected. After determining the MIC, an aliquot was withdrawn from each culture and diluted 100 -fold using YPD medium. Following incubation for $48 \mathrm{~h}$ at $30^{\circ} \mathrm{C}$, the MFC was determined as the lowest concentration of the test compounds in which no recovery of the yeast cells was observed.

\section{Time-kill assay}

The yeast cells grown overnight in YPD medium were diluted into YPD medium to a concentration of $1 \times 10^{6}$ cells $/ \mathrm{mL}$. Dodecanol, dehydrozingerone, or combinations of the drugs were added to the yeast cultures. The treated cell suspensions were incubated at $30^{\circ} \mathrm{C}$ without shaking within $72 \mathrm{~h}$. The measurement of viable cell numbers, based on CFU, was determined after cultivation for $48 \mathrm{~h}$ at $30^{\circ} \mathrm{C}$ on YPD agar plates.

\section{Ribonucleic acid extraction and real-time quantitative reverse transcriptase-polymerase chain reaction}

Total RNA was extracted from the yeast cells using an RNeasy Mini Kit (Qiagen) following the manufacturer's instructions. Briefly, the yeast cells treated with dodecanol, dehydrozingerone, or a combination of the drugs as described above were collected by centrifugation at $5000 \times g$ for $10 \mathrm{~min}$, and then lysed with zymolyase. The RNA samples were purified using the columns provided in the kit, and then DNase treated. The RNA samples were reverse transcribed to generate complementary DNA (cDNA) using ReverTra Ace (TOYO$B O)$. The qRT-PCR was conducted using SsoAdvanced Universal SYBR Green Supermix (Bio Rad), using the cDNA as a template with a CFX Connect Real-Time PCR Detection Systems (Bio Rad). The $20 \mu \mathrm{L}$ qPCR reaction mixtures contained $10 \mu \mathrm{L}$ of $2 \times$ Supermix,

- Table 2 Primer sets for qRT-PCR analysis.

\begin{tabular}{|l|l|}
\hline Gene & Sequence $\left(\mathbf{5}^{\prime} \boldsymbol{\rightarrow} \mathbf{3}{ }^{\prime}\right)$ \\
\hline ACT1-F & CGTCTGGATTGGTGGTTCTATC \\
\hline ACT1-R & GGACCACTTTCGTCGTATTCTT \\
\hline PDR1-F & GGAGCGAAGCTTTTGACAAC \\
\hline PDR1-R & CTGCAGAAATGGTGCTCGTA \\
\hline PDR3-F & GTTTGGGCATGTTTGGACTT \\
\hline PDR3-R & CCCGGTTCAACTTCTTTCAA \\
\hline PDR5-F & ATTCACCAACCCTATGCTATTT \\
\hline PDR5-R & TTACAACCTTCGCCCAAGTC \\
\hline
\end{tabular}

$0.1 \mu \mathrm{L}$ of template, and $5 \mathrm{pmol} / \mu \mathrm{L}$ of both forward and reverse primers. The cycling profile was $3 \mathrm{~min}$ at $95^{\circ} \mathrm{C}$, followed by 40 sequential cycles of $10 \mathrm{~s}$ at $95^{\circ} \mathrm{C}, 30 \mathrm{~s}$ at $55^{\circ} \mathrm{C}$. The relative expression levels of $P D R 1, P D R 3$, and $P D R 5$ were normalized against the expression of housekeeping gene $A C T 1$ as an internal positive control. The primers used in this study are listed in $>$ Table 2.

\section{Efflux of rhodamine $6 \mathrm{G}$}

R6G efflux from the yeast cells was measured by the method of Niimi et al. [30] with slight modifications. Namely, the yeast cells from an overnight culture in YPD medium were centrifuged at $9600 \times g$ for $5 \mathrm{~min}$ at $27^{\circ} \mathrm{C}$. The harvested cells were washed twice with PBS, and resuspended in PBS. The cells were incubated with shaking at $30^{\circ} \mathrm{C}$ for $12 \mathrm{~h}$. The cells suspension was centrifuged at $9600 \times \mathrm{g}$ for $5 \mathrm{~min}$ at $27^{\circ} \mathrm{C}$ and resuspended to a cell density of $5 \times 10^{8}$ cells $/ \mathrm{mL}$ in PBS. R6G was added to a concentration of $10 \mu \mathrm{M}$, and the yeast cells were incubated at $30^{\circ} \mathrm{C}$ for $60 \mathrm{~min}$ to allow uptake of the R6G dye. The R6G-treated cells were washed, and then resuspended at $7.5 \times 10^{7}$ cells $/ \mathrm{mL}$ in PBS. The test compounds and $10 \mathrm{mM}$ glucose were added to the suspension. One $\mathrm{mL}$ aliquots of the suspensions were collected at the indicated times and centrifuged at $2000 \times g$ for $30 \mathrm{~s}$ at $27^{\circ} \mathrm{C}$. The fluorescence intensity in the supernatant was measured with a Tecan GENios microplate reader using $485 \mathrm{~nm}$ excitation and $535 \mathrm{~nm}$ emission filters.

\section{Measurement of reactive oxygen species generation}

Cellular ROS generation was measured using a method dependent on intracellular deacetylation and oxidation of DCFH-DA to produce the fluorescent compound DCF, as described previously [21]. As reported, DCFH-DA as a probe is highly reactive with hydrogen peroxide $\left(\mathrm{H}_{2} \mathrm{O}_{2}\right)$ and may be used in evaluating $\mathrm{ROS}$ generation in yeast cells [21]. After preincubation of the yeast cells $\left(1 \times 10^{7} \mathrm{CFU} / \mathrm{mL}\right)$ in YPD medium with $40 \mu \mathrm{mol} / \mathrm{mL}$ of DCFH-DA at $30^{\circ} \mathrm{C}$ for $60 \mathrm{~min}$, $3.0 \mathrm{~mL}$ aliquots of the cell suspensions were treated with the test compounds for $60 \mathrm{~min}$ at $30^{\circ} \mathrm{C}$, and then were washed and resuspended in $350 \mu \mathrm{L}$ of PBS buffer. The fluorescence intensity of the cell suspension was determined with the Tecan GENios microplate reader (excitation at $480 \mathrm{~nm}$ and emission at $530 \mathrm{~nm}$ ). The arbitrary units assigned to the results were based on the fluorescence intensity of $10^{7}$ cells. $\mathrm{H}_{2} \mathrm{O}_{2}$ was used as a positive control.

\section{Statistical methods}

Statistical evaluation was performed using Student's t-test to compare the results between experimental groups. A $p$-value $<0.05$ was considered statistically significant.

\section{Acknowledgements}

This work was partly funded by the Japan Society for the Promotion of Science, Grants-in-Aid for Scientific Research (C) 25460128 and 16 K08299.

\section{Conflict of Interest}

No conflict of interest has been declared by the authors. 


\section{References}

[1] Miceli MH, Díaz JA, Lee SA. Emerging opportunistic yeast infections. Lancet Infect Dis 2011; 11: 142-151

[2] Fairlamb AH, Gow NA, Matthews KR, Waters AP. Drug resistance in eukaryotic microorganisms. Nature Microbiol 2016; 1: 16092

[3] Masiá Canuto M, Gutiérrez Rodero F. Antifungal drug resistance to azoles and polyenes. Lancet Infect Dis 2002; 2: 550-563

[4] Polak A, Hartman PG. Antifungal chemotherapy-are we winning? Prog Drug Res 1991; 37: 181-269

[5] Gonçalves SS, Souza ACR, Chowdhary A, Meis JF, Colombo AL. Epidemiology and molecular mechanisms of antifungal resistance in Candida and Aspergillus. Mycoses 2016; 59: 198-219

[6] Ghannoum MA, Rice LB. Antifungal agents: Mode of action, mechanisms of resistance, and correlation of these mechanisms with bacterial resistance. Clin Microbiol Rev 1999; 12: 501-517

[7] Li XZ, Nikaido H. Efflux-mediated drug resistance in bacteria: An update. Drugs 2009; 69: 1555-1623

[8] Cannon RD, Lamping E, Holmes AR, Niimi K, Baret PV, Keniya MV, Tanabe K, Niimi M, Goffeau A, Monk BC. Efflux-mediated antifungal drug resistance. Clin Microbiol Rev 2009; 22: 291-321

[9] Paul S, Moye-Rowley WS. Multidrug resistance in fungi: Regulation of transporter-encoding gene expression. Front Physiol 2014; 5: 143

[10] Lu WD, Qin Y, Yang C, Li L, Fu ZX. Effect of curcumin on human colon cancer multidrug resistance in vitro and in vivo. Clinics (Sao Paulo) 2013; 68: 694-701

[11] Tang XQ, Bi H, Feng JQ, Cao JG. Effect of curcumin on multidrug resistance in resistant human gastric carcinoma cell line SGC7901/ VCR. Acta Pharmacol Sin 2005; 26: 1009-1016

[12] Si M, Zhao J, Li X, Tian JG, Li YG, Li JM. Reversion effects of curcumin on multidrug resistance of MNNG/HOS human osteosarcoma cells in vitro and in vivo through regulation of P-glycoprotein. Chin Med J 2013; 126: 4116-4123

[13] Sharma M, Manoharlal R, Shukla S, Puri N, Prasad T, Ambudkar SV, Prasad R. Curcumin modulates efflux mediated by yeast $A B C$ multidrug transporters and is synergistic with antifungals. Antimicrob Agents Chemother 2009; 53: 3256-3265

[14] Tatsuzaki J, Bastow KF, Nakagawa-Goto K, Nakamura S, Itokawa H, Lee $\mathrm{KH}$. Dehydrozingerone, chalcone, and isoeugenol analogues as in vitro anticancer agents. J Nat Prod 2006; 69: 1445-1449

[15] Fujita K, Ishikura T, Jono Y, Yamaguchi Y, Ogita A, Kubo I, Tanaka T. Anethole potentiates dodecanol's fungicidal activity by reducing PDR5 expression in budding yeast. Biochim Biophys Acta 2017; 1861: 477-484

[16] Hampannavar GA, Karpoormath R, Palkar MB, Shaikh MS. An appraisal on recent medicinal perspective of curcumin degradant: Dehydrozingerone (DZG). Bioorg Med Chem 2016; 24: 501-520
[17] Kubra IR, Murthy PS, Rao LI. In vitro antifungal activity of dehydrozingerone and its fungitoxic properties. J Food Sci 2013; 78: M64-M69

[18] Salin H, Fardeau V, Piccini E, Lelandais G, Tanty V, Lemoine S, Jacq C, Devaux F. Structure and properties of transcriptional networks driving selenite stress response in yeasts. BMC Genomics 2008; 9: 333

[19] Sipos G, Kuchler K. Fungal ATP-binding cassette (ABC) transporters in drug resistance \& detoxification. Curr Drug Targets 2006; 7 : 471-481

[20] Ma M, Liu ZL. Comparative transcriptome profiling analyses during the lag phase uncover YAP1, PDR1, PDR3, RPN4, and HSF1 as key regulatory genes in genomic adaptation to the lignocellulose derived inhibitor HMF for Saccharomyces cerevisiae. BMC Genomics 2010; 11: 660

[21] Fujita K, Tatsumi M, Ogita A, Kubo I, Tanaka T. Anethole induces apoptotic cell death accompanied by reactive oxygen species production and DNA fragmentation in Aspergillus fumigatus and Saccharomyces cerevisiae. FEBS ] 2014; 281: 1304-1313

[22] Yogosawa S, Yamada Y, Yasuda S, Sun Q, Takizawa K, Sakai T. Dehydrozingerone, a structural analogue of curcumin, induces cell-cycle arrest at the G2/M phase and accumulates intracellular ROS in HT-29 human colon cancer cells. J Nat Prod 2012; 75: 2088-2093

[23] Cuenca-Estrella M, Gomez-Lopez A, Mellado E, Monzon A, Buitrago M], Rodriguez-Tudela JL. Activity profile in vitro of micafungin against Spanish clinical isolates of common and emerging species of yeasts and molds. Antimicrob Agents Chemother 2009; 53: 2192-2195

[24] Yutani M, Hashimoto Y, Ogita A, Kubo I, Tanaka T, Fujita K. Morphological changes of the filamentous fungus Mucor mucedo and inhibition of chitin synthase activity induced by anethole. Phytother Res 2011; 25: $1707-1713$

[25] Fujita K, Fujita T, Kubo I. Anethole, a potential antimicrobial synergist, converts a fungistatic dodecanol to a fungicidal agent. Phytother Res 2007; 21: 47-51

[26] Anuchapreeda S, Leechanachai P, Smith MM, Ambudkar SV, Limtraku PN. Modulation of P-glycoprotein expression and function by curcumin in multidrug-resistant human KB cells. Biochem Pharmacol 2002; 64: 573-582

[27] Choi BH, Kim CG, Lim Y, Shin SY, Lee YH. Curcumin down-regulates the multidrug-resistance mdr1b gene by inhibiting the PI3K/Akt/NFKB pathway. Cancer Lett 2008; 259: 111-118

[28] Fujita K, Kubo I. Antifungal activity of octyl gallate. Int J Food Microbiol 2002; 79: 193-201

[29] Kubo I, Fujita K, Kubo A, Nihei K, Lunde CS. Modes of antifungal action of (2E)-alkenals against Saccharomyces cerevisiae. J Agric Food Chem 2003; 51: 3951-3957

[30] Niimi M, Niimi K, Takano Y, Holmes AR, Fischer F], Uehara Y, Cannon RD. Regulated overexpression of CDR1 in Candida albicans confers multidrug resistance. J Antimicrob Chemother 2004; 54: 999-1006 